\title{
SUTURELESS AORTIC VALVE REPLACEMENT: DOES IT MAKE SENSE?
}

\author{
Kenan Sever ${ }^{1}$, Oguz Konukoglu ${ }^{1}$, Ozgur Yildirim ${ }^{1}$, Hakan Kilercik ${ }^{1}$, and Denyan \\ Mansuroglu ${ }^{1}$
}

${ }^{1}$ Yeni Yuzyil University Gaziosmanpaşa Hospital

October 8, 2020

\begin{abstract}
BACKGROUND Aortic valve stenosis is the most common valve disease in the elderly. Sutureless aortic valve replacement has been introduced as an alternative to conventional AVR in elderly high-risk patients. The aim of this study is reporting our single-center experience regarding early outcomes. METHODS Between December 2014 and December 2019,91 patients (45 Women, 46 Males) were undergone aortic valve replacement in our clinic (49 Sutureless, 42 conventional). Perioperative clinical and echocardiographic outcomes were assessed in all patients. RESULTS The average age was $73.08 \pm 7.53$ and $66.26 \pm 8.63$ for sutureless and conventional groups. The mean cross-clamp time and the cardio- pulmonary bypass (CPB) time were 72.86 and $91.88 \mathrm{~min}$, and 104.96 and $119.81 \mathrm{~min}$, respectively. In sutureless group, 30 (61.2\%) patients underwent additional procedures. These were CABG, mitral interventions, tricuspid repair, ascending aortic surgery and myxoma. Preoperative peak and mean pressure gradients decreased from 78.16 and $48.95 \mathrm{mmHg}$ to 17.47 and $10.06 \mathrm{mmHg}$ postoperatively for sutureless group. It was 71.53 and 43.89 to 29.16 and 15.14 for conventional group. Paravalvular leak and permanent pace-maker requirement due to AV-block rates were $6.1 \%$. The mean ICU stay were 3.69 and 2.31 days, mean hospital stay were 10.08 and $8.62,30$-day overall mortality rates were $8.2 \%$ and $4.8 \%$ for sutureless and conventional groups. CONCLUSION The evaluation of our experience suggests that sutureless aortic valve replacement has advantages in terms of shorter cross-clamp and CPB duration, and postoperative aortic gradients. Its benefits could be more prominent in complex cases or minimally invasive surgery
\end{abstract}

\section{SUTURELESS AORTIC VALVE REPLACEMENT: DOES IT MAKE SENSE?}

Kenan Sever ${ }^{1}$ Ass. Prof. Dr., Oguz Konukoglu ${ }^{1}$ Ass. Prof. Dr., Ozgur Yildirim ${ }^{1}$ Ass.Prof Dr.., Hakan Kilercik ${ }^{2}$ Ass. Prof. Dr, Denyan Mansuroglu ${ }^{1}$ Prof. Dr.

1-Istanbul Yeni Yuzyil University Gaziosmanpasa Hospital, Cardiovascular Surgery Department

2-Istanbul Yeni Yuzyil University Gaziosmanpasa Hospital, Anesthesiology Department

Corresponding Author: Oguz Konukoglu

Adress: Yeni Yüzyıl Üniversitesi, Özel Gaziosmanpaşa Hastanesi, Merkez, Çukurçeşme Cd. No:51, 34245 Gaziosmanpaşa/İstanbul, TURKEY

e-mail:oguzkonukoglu@yahoo.com

Telephone: 00905058689732

Fax: 00902126153849

ABSTRACT

BACKGROUND 
Aortic valve stenosis is the most common valve disease in the elderly. Sutureless aortic valve replacement (AVR) has been introduced as an alternative to conventional AVR in elderly high-risk patients. This study aims to report our single-center experience regarding early outcomes.

\section{METHODS}

Between December 2014 and December 2019, 91 patients (45 women, 46 men) underwent aortic valve replacement at our clinic (49 sutureless, 42 conventional). The inclusion criteria were severe symptomatic valve disease, New York Heart Association (NYHA) class II or higher, and age $>55$ years. Perioperative clinical and echocardiographic outcomes were assessed in all patients.

\section{RESULTS}

The average age was $73.08 \pm 7.53$ years in the sutureless group and $66.26 \pm 8.63$ years in the conventional group. The mean cross-clamp and cardiopulmonary bypass (CPB) times were 72.86 and $91.88 \mathrm{~min}$, respectively, in the sutureless group, and 104.96 and $119.81 \mathrm{~min}$, respectively, in the conventional group. In the sutureless group, $30(61.2 \%)$ patients underwent additional procedures such as CABG, mitral interventions, tricuspid repair, ascending aortic surgery, and myxoma removal. Preoperative peak and mean pressure gradients decreased from 78.16 and $48.95 \mathrm{mmHg}$ to 17.47 and $10.06 \mathrm{mmHg}$ postoperatively in the sutureless group, and from 71.53 and $43.89 \mathrm{mmHg}$ to 29.16 and $15.14 \mathrm{mmHg}$ in the conventional group. Paravalvular leak and permanent pacemaker requirement due to AV-block rates were $6.1 \%$. The mean ICU stay was 3.69 and 2.31 days, the mean hospital stay was 10.08 and 8.62 days, and the 30 -day overall mortality rates were $8.2 \%$ and $4.8 \%$ in the sutureless and conventional groups, respectively.

\section{CONCLUSION}

The evaluation of our experience suggests that sutureless aortic valve replacement has advantages in terms of shorter cross-clamp time, reduced CPB duration, and postoperative aortic gradients. Its benefits could be more prominent in complex cases or minimally invasive surgery.

Keywords: sutureles, aortic valve, intuity, perceval

There is no conflict of interest about the authors of this study.

Aortic valve stenosis is the most common valve disease in an aging population, and the annual mortality expectancy in patients with severe stenosis is $30-50 \% .^{1,2}$ The most effective treatment for severe aortic valve stenosis is aortic valve replacement (AVR). Clinical studies show improved left ventricular systolic and diastolic function due to the regression of left ventricular hypertrophy after aortic valve replacement. ${ }^{3}$ Biologic aortic valves are preferred to reduce postoperative gradient levels and avoid complications related to warfarin use in elderly patients with aortic stenosis. Clinical reports indicate a low gradient and long-term durability with pericardial and porcine valves, with good results up to 20 years. ${ }^{4}$ However, these valves are implanted on a stent with a Dacron graft, and a residual gradient (depending on graft bending) may occur in a narrow and calcified annulus. Bioprosthetic valves without stents have been developed to overcome these problems and provide a greater orifice area. ${ }^{5}$ The advantage of the stent-free structure is that it increases the effective valve area and decreases the gradient. However, their implantation is more difficult compared to the stented valves and leads to increased surgical cross-clamp time. ${ }^{6}$ Transcatheter-mediated aortic valve implantation (TAVI) is recommended in the elderly and high-risk patients; however, studies have shown that TAVI has high rates of complications. ${ }^{7}$ Sutureless aortic prosthetic valves developed in recent years maximize effective valve orifice area and reduce cross-clamp time. ${ }^{8,9}$ In the sutureless implantation technique, in addition to conventional AVR procedures, the valve is placed in a sutureless fashion or positioned with three stitches, resulting in a significant reduction in cross-clamp time. ${ }^{10}$ These valve prostheses have been used in our country since 2012. This study aims to discuss the short-term results of 49 sutureless AVR cases and compare them with conventional AVR.

MATERIALS AND METHODS 
Between December 2014 and December 2019, 49 patients (30 women, 19 men) received sutureless aortic valves, and 42 patients underwent conventional aortic valve replacement at our clinic by the same surgeon. The inclusion criteria were severe symptomatic valve disease, New York Heart Association (NYHA) class II or higher, and age $>55$ years. All patients received informed consent forms. The necessary permissions were obtained for the study from the ethics committee of our university.

Two types of sutureless valves can be used with the diagnosis of severe aortic valve disease in our country: Perceval S (Sorin Biomedica Cardio Srl, Sallugia, Italy) valves, and Edwards Intuity (Edwards Lifesciences, Irvine, CA, USA) valves. We used 16 Sorin Perceval valves (32.7\%) and 33 Edwards Intuity valves (67.3\%) for our patient groups.

A median sternotomy was performed on most patients following general anesthesia and orotracheal intubation. Unicaval venous and aortic arterial cannulation was applied to isolated cases. Bicaval venous cannulation was used in complicated cases such as concomitant tricuspid or mitral interventions. The aorta was split from the pulmonary artery. Transverse/oblique aortotomy was performed after placing the cross-clamp. The heart was arrested by applying antegrade isothermic blood cardioplegia from the coronary ostia. The process was repeated at intervals of 20 minutes until the aortotomy was closed. In complex cases, cardiac protection was achieved by retrograde cardioplegia. The aortic valve was excised, and the annulus decalcified. The valve size was measured using original valve scales.

For Sorin Perceval valve implantation, a transverse aortotomy was performed about $2 \mathrm{~cm}$ above the sinotubular junction. After excision and decalcification, the valve applicator was advanced to the aortic position. The valve was expanded after verifying the appropriate positioning of the prosthesis. The applicator was taken out. A post dilatation balloon was inserted in the aortic valve and dilated for 30 seconds at a pressure of 4 atmospheres.

For Edwards Intuity valve implantation, an oblique aortotomy was performed about $1 \mathrm{~cm}$ above the sinotubular junction. Following excision and decalcification, three 4-0 prolene sutures were placed at the nadir of the aortic sinus. The valve was prepared by washing in saline solution for 2 minutes. The balloon inflator was filled with a $40 \mathrm{ml}$ saline solution, and a balloon connection was made. The sutures (previously passed through the commissures) were unified by snares and passed through the sewing ring of the lid. The guide sutures were tightened to advance until the valve was placed in the exact aortic position with the help of the valve applicator. The snares were squeezed, and the valve was fixed to its position. The balloon inflation was achieved at pressures ranging from 3 to 5 atmospheres for at least 10 seconds, and the stabilizer ring under the valve was expanded. The guide sutures were firmly tied above the valve annular ring, and the valve applicator was taken out.

Following valve implantation, the aortotomy was closed with 4-0 or 5-0 prolene continuous sutures. After the cross-clamp was removed, the cardiopulmonary bypass was terminated following standard procedures, and epicardial pacemaker wires were routinely placed.

In the presence of coronary bypass graft surgery (CABG), distal bypasses were done before valve implantation and proximal bypasses after aortic valve implantation under cross-clamping.

In the presence of additional mitral and tricuspid valve intervention, atriotomy and valve procedures were performed before aortic valve implantation.

Valve position and possible leakage after implantation were evaluated with intraoperative TEE in all patients. All patients underwent transthoracic echocardiography before hospital discharge.

Demographic data, preoperative, and postoperative parameters were compiled by retrospectively accessing the data of all patients undergoing aortic valve implantation.

SPSS Ver. 22.0 (SPSS Inc., Chicago, IL, USA) was used in data analysis. Average and standard deviation for numerical evaluations and percentage values for categorical variables were calculated. For categorical variables, the results were compared with the chi-square test. The numerical groups were compared with 
paired-samples t-testing. For non-parametric groups, groups were compared with independent samples test (Mann-Whitney U). All p-values $<0.05$ were considered to indicate statistical significance.

\section{RESULTS}

Between December 2014 and December 2019, 49 patients (30 [61.2\%] females, 19 [38.8\%] males) underwent sutureless AVR valve implantation at our clinic. The average age was $73.08+-7.53$ years. Table 1 shows the demographic information.

In addition to sutureless AVR, $30(61.2 \%)$ patients underwent additional procedures such as CABG, mitral interventions, tricuspid repair, ascending aortic surgery, and myxoma removal. (Table 2) Sorin Perceval S was used in 16 patients $(32.7 \%)$ and Edwards Intuity in $33(67.3 \%)$ patients. The average cardiopulmonary bypass time was $104.96+-41.63 \mathrm{~min}$. ( $76.47+-24.79 \mathrm{~min}$. in isolated cases), and the average cross-clamp time was $72.86+-34.09 \mathrm{~min}$. (51.05+-21.68 min. in isolated cases)(Table 3). The average erythrocyte suspension requirement was $3.90+-2.85$ units in the sutureless group (3.42+-1.77 units in isolated cases). The hospitalization time in the intensive care unit was $3.73+-6.20$ days, and the discharge time was $10.08+-$ 6.56 days. (Table 5) Three patients underwent revision for bleeding.

Preoperative and postoperative echocardiographic data are shown in Table 4. The preoperative and postoperative ejection fractions were $54.77+-10.90$ and $55.87+-9.87$, respectively $(\mathrm{p}=0.313)$. The preoperative and postoperative maximum aortic gradients were $78.16+-30.24$ and $17.47+-6.16$, respectively $(\mathrm{p}=0.000)$; the preoperative and postoperative mean aortic gradients were $48.95+-21.28$ and $10.06+-3.63$, respectively $(\mathrm{p}=0.000)$.

In one case, the patient received a Bentall operation due to aortic root rupture. In this case, death occurred in the ICU period.

Aortic valve insufficiency $>2$ deg was seen in 4 patients. One patient died in the ICU. Two patients underwent reoperation for heart failure symptoms, and one of them died after the second operation. Aortic valve insufficiency $>2 \mathrm{deg}$ was zero in the conventional control group.

Postoperative AV block that required permanent pacemaker occurred in $3(6.1 \%)$ patients.

Cumulative short-term mortality was $8.2 \%$ (4 patients) in the sutureless group and $4.8 \%$ (2 patients) in the conventional control group, $\mathrm{p}=0.683$.

\section{DISCUSSION}

Aortic stenosis is most common in western countries with an aging population. ${ }^{1}$ The increase in high-risk patients has led to the development of less invasive treatment options and an increasing number of cases. ${ }^{11}$ Currently, TAVI and sutureless aortic valve replacement are highlighted as less invasive methods. ${ }^{2}$

Sutureless valves showed better hemodynamic results in our series in terms of gradient change (preoperative gradient-postoperative gradient) after implantation. After the operation, the maximum gradient change was $60.68 \mathrm{mmHg}$ in the sutureless group and $49.74 \mathrm{mmHg}$ in the conventional group $(\mathrm{p}=0.003)$. The mean gradient change was $38.88 \mathrm{mmHg}$ in the sutureless group and $28.74 \mathrm{mmHg}$ in the conventional group $(\mathrm{p}=0.004)$. (Table 5) Studies have shown that sutureless aortic valves reveal a stable reduction of gradients postoperatively. ${ }^{3,11,12} \mathrm{D}^{\prime}$ Onofrio et al. observed that transapical and sutureless valves have lower mean aortic gradients than conventional aortic valve replacements. ${ }^{11}$

The sutureless implantation technique is associated with a reduction in cross-clamp and total perfusion times, which are independent risk factors. ${ }^{13}$ A study by Flameng et al. found that the average cross-clamp and total perfusion times were 22 and 46 minutes, respectively, in selected cases. ${ }^{14}$ In a meta-analysis compiled by twelve studies, isolated AVR times were 33 and 57 minutes, and the average cross-clamp and total perfusion times for conventional isolated AVR cases in the STS knowledge base were 78 and 106 minutes, respectively. ${ }^{2}$ Cross-clamp and total perfusion times for isolated AVR in our patient group were 51 and 76 minutes, respectively. 
In our patient group, 30 patients $(61.2 \%)$ underwent additional procedures such as CABG (51\%), ascending aortic procedure (8.2\%), MVR (6.1\%), and other operations. Cross-clamp and total perfusion times were 72 and 104 minutes, respectively, in patients undergoing additional procedures in the sutureless group.

Paravalvular aortic regurgitation is seen more commonly in sutureless than in conventional aortic valve replacements. ${ }^{2-4,15,16}$ Our study group showed moderate/severe regurgitation in $3(6.1 \%)$ patients and no regurgitation in conventional cases $(\mathrm{p}=0.121)$. The AV block is a known complication. ${ }^{2,10,12,15,16}$ Three patients developed permanent AV conduction blocks requiring pacemaker implantation. We did not see permanent AV blocks in the conventional group.

Mini sternotomy provides procedural and exposure advantages in isolated AVR cases. Although we performed sternotomy in most of our patients, we preferred the upper mini sternotomy in our last cases for isolated sutureless AVR.

Although we started our learning curve with Edwards Intuity, currently, we prefer to use the Sorin Perceval S valve for its design. The first handicap of the Intuity valve is the wide and rigid collar under the valve. This extra-anatomic position can cause stretching of the mitral valve. One of our patients experienced aortic root rupture following blunt trauma due to the inflation of the collar. The patient received a Bentall operation and died eventually. The second handicap can be seen in late re-operations with the Intuity valve. It can be highly challenging to detach the valve in cases of infra-aortic and possible mitral adhesion of the collar.

Factors that achieve success in sutureless valve implantation based on our experience are as follows: 1) Not to make too much valve resection; 2) Appropriate sizing; 3) Avoiding barotrauma while inflating the balloon.

Mean ICU stay times were longer in the sutureless group (3.69 days in sutureless, 2.31 in conventional, $\mathrm{p}=0.969$ ) and mean hospital stay was also higher (10.02 days in sutureless, 8.68 in conventional, 0.855). Although there was no statistically significant difference between ICU and hospital stay times, more than one day of mean stay in the ICU could influence the costs. Also, the price of the sutureless valves is around three-fold that of the bioprosthetic valves in our country.

We did not observe mortality in the isolated sutureless AVR group (19 patients). In a different series, isolated AVR groups had short-term mortality that could be considered satisfactory, around $3 \%$. We observed a mortality rate of $13.3 \%$ (4 patients) among 30 non-isolated cases. Of these, two patients received reoperations. In a multi-centric study conducted in Europe, the mortality rate was reported as $12.9 \%$ on an average in non-isolated sutureless AVR cases. ${ }^{17}$

A mortality rate of $8.2 \%$ (4 patients) was observed in the sutureless group of our study. The mortality rate in the conventional group was $4.8 \%$ (2 patients). However, no significant difference was detected between the sutureless and conventional groups $(\mathrm{p}=0.683)$. EuroSCORE 2 values of the sutureless group were significantly higher (5.51+-7.29 for sutureless, $2.80+-3.52$ for the conventional group, $\mathrm{p}=0.01)$. It showed that a sutureless strategy was preferred in complicated cases to reduce cross-clamp times. In previous studies on conventional aortic replacement, the mortality rate was $4-10 \%$, and factors such as advanced age, low ejection fraction, renal insufficiency, and severe aortic calcification were cited among the causes that influenced mortality. ${ }^{18}$

Thus, sutureless valves offer better hemodynamic and intraoperative results such as lower postoperative aortic gradient and shorter cross-clamp and cardiopulmonary bypass times. However, sutureless valve selection did not make a significant change in terms of hospital stay, ICU stay, and mortality. These variables were also higher in the sutureless group. Although it is not easy to clearly state whether or not these handicaps are directly related to the sutureless technique, we could not find convincing results that reveal superiority to conventional aortic replacement.

Sutureless valve technology has brought new dimensions to aortic valve replacement. It has unique advantages and unique complications. The benefits could be enhanced in presence of additional procedures or minimally invasive strategies with acceptable results.

LIMITATIONS OF THE STUDY 
One limitation of this study was that it was based on data from a single center with a limited number of patients. The study revealed early outcomes, and it is necessary to have more data documenting long-term results. Although age and patient characteristics were alike, the EuroSCORE 2 values for the sutureless group were higher than those for the control group.

\section{CONFLICTS OF INTEREST}

There is no conflict of interest relevant to this article.

\section{Hosted file}

Table 1.pdf available at https://authorea.com/users/365247/articles/485431-sutureless-aorticvalve-replacement-does-it-make-sense

\section{Hosted file}

Table 2.pdf available at https://authorea.com/users/365247/articles/485431-sutureless-aorticvalve-replacement-does-it-make-sense

\section{Hosted file}

Table 3.pdf available at https://authorea.com/users/365247/articles/485431-sutureless-aorticvalve-replacement-does-it-make-sense

\section{Hosted file}

Table 4.pdf available at https://authorea.com/users/365247/articles/485431-sutureless-aorticvalve-replacement-does-it-make-sense

\section{Hosted file}

Table 5.pdf available at https://authorea.com/users/365247/articles/485431-sutureless-aorticvalve-replacement-does-it-make-sense 